\title{
Modular sensor architecture for unobtrusive routine clinical diagnosis*
}

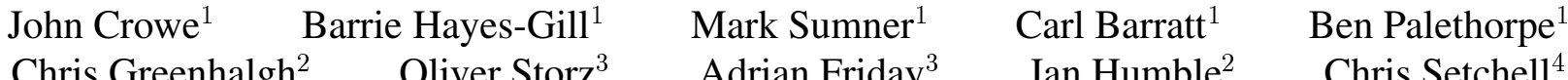 \\ Cliff Randell ${ }^{4} \quad$ Henk L Muller ${ }^{4}$ \\ ${ }^{1}$ University of Nottingham, School of Electrical and Electronic Engineering \\ ${ }^{2}$ University of Nottingham, Department of Computer Science \\ ${ }^{3}$ University of Lancaster, Department of Computer Science \\ ${ }^{4}$ University of Bristol, Department of Computer Science
}

\begin{abstract}
Clinical diagnosis of pathological conditions is accomplished regularly via the recording and subsequent analysis of a physiological variable from a subject. Problems with current common practice centre around the obtrusive and rigid nature of this process. These include the length, timing and location of the diagnostic recording session, transfer of data to clinical staff, liaison between clinical staff and subjects and the integration of such diagnostic check-ups into the overall health care process.

We have designed a modular diagnostic monitor that is centered around a wearable computer system which, when integrated into a suitable computer network and database architecture, is capable of addressing the above problems. The system is modular, allowing researchers and practitioners to utilise various sensor modules, reconfigure the unit in terms of its on-board storage and wireless telemetry capabilities, select the appropriate level of data preprocessing (before archiving data) and choose the appropriate level and nature of feedback to the subject. The system is GRID enabled, supporting e-clinical-trials. GRID clients can display live data, historical data, or perform data mining.
\end{abstract}

\section{Introduction}

Several decades ago clinical diagnosis would have required both patient and practitioner being in the same room at the same time. The advent of small, low-powered and robust electronic and microprocessor systems around the 1970 's led to the possibility of monitoring patients during their normal (working) day. The most common example is

* Funding for this work is received from the U.K. Engineering and Physical Sciences Research Council, Grant Numbers. GR/N15986/01 and GR/R85877/01 the portable ECG (commonly known as a Holter monitor; named after a pioneer of this field) with similar units also existing for (intermittent) blood pressure monitoring and other physiological variables.

However, these devices still possess a number of drawbacks. Firstly, such devices typically usually log only one stream of physiological data that has no contextual setting. However, contextual data (for example in its most basic form of the time, location and activity of the subject) can be useful in explaining health patterns. Secondly most commercially available units have a fundamental design constraint in that they are designed to log data for a fixed period at the end of which the data will be downloaded for analysis. Therefore they cannot be used to inspect data on the fly, and to issue requests to the patient to perform certain activities or manoeuvres for example.

The widespread availability of wireless communication networks in the developed world and computer systems networks plus the decreasing size and power consumption of electronics coupled with advances in battery technology means that the production of a new generation of diagnostic monitoring equipment is now within reach.

Advantages are a wider choice (on a plug-and-play basis) of both physiological and contextual (e.g. location, activity and environmental conditions) sensors under computer control. The flexibility offered include the ability to determine adaptively the level of processing and compression of data before storage and / or telemetry and provide a mechanism for feedback to the subject as and when appropriate.

The scenario that we use our medical jacket for is that the patient is wired up and can subsequently walk around. Any time that the jacket comes in reach of a wireless accesspoint data is offloaded onto the GRID. A practitioner can access this data over the GRID. In particular, they can correlate physical activity (walking on a hill at a certain speed, information provided by the GPS) with medical recordings (heart activity, Oxygen saturation, collected by ECG and 
$\mathrm{SpO} 2$ sensors). This scenario allows patients to be monitored in their every environment, rather than in an artificial environment (a treadmill in a hospital).

\section{Background}

We have a long standing interest in the recording of physiological data for clinical diagnosis $[2,4,5,9,11]$ that has included the use of wireless telemetry $[1,13]$ and adoption of sophisticated signal processing strategies for bio-signal processing $[6,8]$. In addition we have widespread experience in the use of wearable computers [12] for the interactive dissemination of information and entertainment plus the linking of such technology into computer networks for the filtering, archiving, processing and efficient retrieval of data.

Currently we are collaborating in a UK government funded project entitled "Grid Based Medical Devices for Everyday Health" [16] in which one of the aims is to use our collective experience to produce the type of unobtrusive monitor outlined above. In addition we are involved in a related environmental monitoring Grid based project [15] plus a recently funded multi-centre UK Government programme, MATCH, concerned with the development of formal tools for Health Technology assessment.

Our close working links with clinical colleagues, staff in the IT departments of hospitals and the medical device industry means that we are operating with full knowledge of the problems associated with the introduction of new technology into the healthcare sector (e.g. reimbursement issues, strictly controlled access to hospital networks, patient confidentiality, clinical trial protocols etc.)

\section{Unobtrusive Diagnostic Monitoring}

We have used a standard wearable platform (the Cyberjacket), which comes with wireless connectivity, and augmented it with three purpose built sensors: an ECG, a Oxygen Saturation Monitor and a temperature sensor. This augments the standard sensors on the Cyberjacket for position sensing (using GPS), and motion sensing (using accelerometers). The nature of the Cyberjacket means that the system is completely modularised. This means that health researchers can easily customise a jacket for their experiment. It also decreases the cost of units, as they are reusable in different configurations

\subsection{Wearable architecture}

The wearable system that we use is the Cyberjacket [12] developed at Bristol. It consists of an ADS-Bitsy processor unit (based on the StrongARM), with a custom 9-wire bus

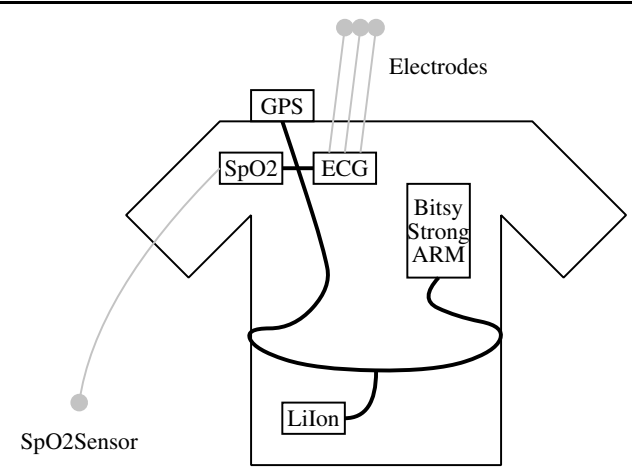

Figure 1. Jacket structure

embedded in the fabric of the jacket. Sensors can be connected anywhere to the bus. On the bus, we have power, ground, and three serial links, each running at different speeds and different voltage levels. Two links are running at RS-232 levels for dedicated RS-232 devices and can be run at any speed. Any commercially available device that runs RS-232 (such as a GPS) can be connected to one of those busses. The third bus runs at TTL level, at 4800 baud. It is used as a drop-link bus on which several devices can be connected (we have tried up to 20 devices). This shared bus has a defined request-post protocol and a contention line to allow unsolicited data to be posted.

The reason why we have employed a bus-based system is because of power distribution issues. In our present system we have one or more central batteries that power all components of our system (sensors, computation, communication). Changing batteries in our Cyberjacket is straightforward as we just need to change the central battery. We are investigating non bus based solutions based on various wireless protocols, but this will involve powering all components individually, making battery changing a painstaking operation. With one central battery the whole system runs out of power at the same time. With $n$ distributed batteries each powering a part of the system, each battery will run out separately, resulting in an $n$-fold decrease in the Mean Time Between Failure (MTBF) of the system as a whole.

We have employed an RS-232 based bus in favour over something more modern such as the USB bus. An RS232 bus allows us to straightforwardly design a drop-link. Adding devices at any place on our bus involves clipping a connector on the bus, nothing more. If a a star network is employed instead of a drop link, then new wires have to be routed for any sensor added. An example layout is shown in Figure 1.

Standard sensors that are available on the Cyberjacket include accelerometers, GPS, indoor location detection and compasses. Accelerometers and compasses can be attached to different places to measure different activities. For ex- 

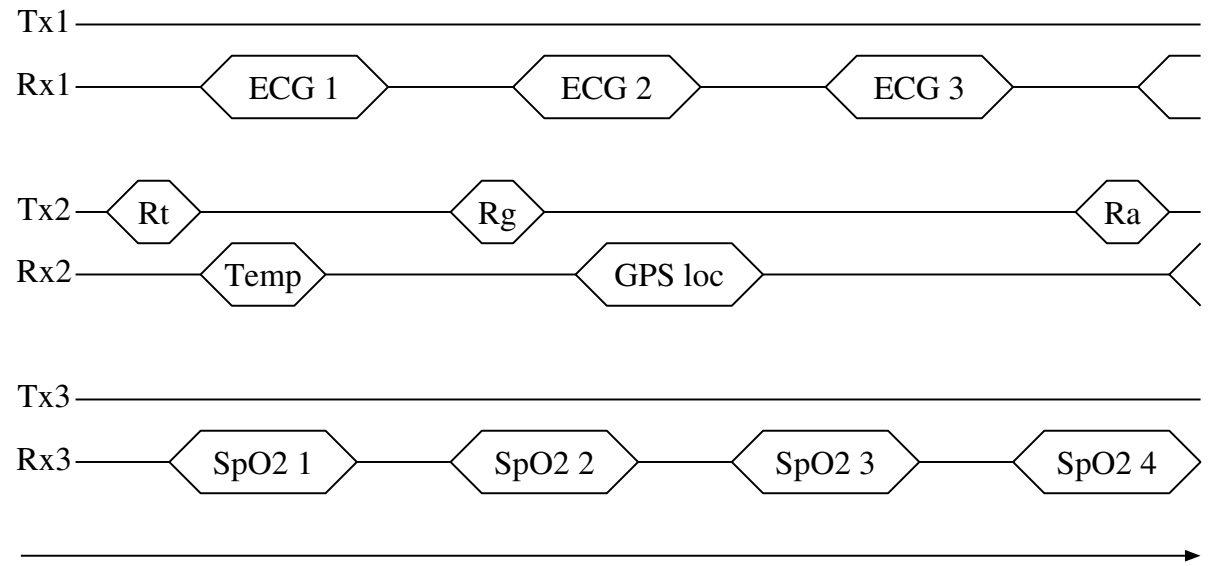

Time $\rightarrow$

Figure 2. The protocol over the three busses

ample, a compass mounted on a pair of headphones measures the direction in which the wearer is looking, and an accelerometer mounted vertically on a subject's back can detect walking. In addition to the serial buses, the ADS-Bitsy has stereo sound $\mathrm{I} / \mathrm{O}$ that can be used to give feedback to the wearer.

\subsection{Sensor architecture}

Typically, information is gathered from multiple sensor types in order to accurately track and diagnose a given disease state. The combination of electro-physiological and other medical parameters that must be monitored for a specific medical condition varies widely, subsequently the sensors used in this study are designed to ease their introduction and withdrawal from the monitoring exercise as necessary. At the time of writing, the sensors integrated include:

Blood oxygen saturation sensor (SpO2) : derives the level of oxygen present in arterial blood via a calculation on the ratio of light absorption resulting from oxygenated and reduced haemoglobin through a well-perfused body part (eg the finger).

Electrocardiogram (ECG) : signal produced on the surface of the skin by the electrical activity of the heart.

Skin temperature : monitored using clinical grade thermistors.

Each sensor uses analogue techniques to obtain, noninvasively, signals that impart rich medical information about the patient. From here, these signals are conditioned, sampled and processed prior to assembly into packets of digitised data. All sensors conform to a packet format, which includes a header (depicting the origin of the packet and the type of data included within it), the data itself and some indication on the validity of the data.

The packets of data generated by a particular sensor can then be time-division multiplexed with those produced by other sensors, or allocated to a dedicated channel depending on the quantity of data produced, as shown in Figure 2. To give an example, output from the ECG sensor is in standard RS232 serial format in packets which begin with the ASCII sequence $\$ S E 0$ (where $\mathrm{S}=$ streaming, $\mathrm{E}=\mathrm{ECG}, 0$ $=$ minor sensor number) and carry a payload of 100 bytes, transmitted at 19200 baud.

\subsection{Output: the GRID}

Conceptually, sensor data that is collected on the wearable is available on the Internet as a GRID service. The information is pushed into a database, and a practitioner can query the database. They can either view data in real time (with a slight lag due to the GRID), or they can scroll back and view old information [3]. In order to implement this, we needed to overcome a couple of difficulties. We opted to use an 802.11 wireless network interface because of its low operational cost. 802.11 cells are installed in strategic places, for example near a hospital (if the wearer is sent for a walk around the hospital), or in the wearer's office and home if someone is monitored continuously. Because the wireless connection is present only intermittently, the sensor data is cached on the Bitsy, and off-loaded when a wireless connection becomes available (transparent to wearer and practitioner). When the data is sent off, it is sent to a proxy on the Internet, where it is made available as a GRID-service.

The proxy supports a flexible device interrogation protocol based on the Sensor Modelling Language (SensorML) [10] that allows sensors on the wearable to be dynamically discovered and deployed onto the Grid. We use the 
Global Toolkit version 3 (GT3) [7] to present data from the wearable as Open GRID Service Architecture (OGSA) [14] compliant services. The system has been designed in an overtly modular fashion allowing repurposing of the sensor data in a range of Grid based medical applications.

In our system we provide two main interfaces to the data: a clinician's interface and a management interface allowing data to be passed on to other Grid services and applications. Significantly, the system incorporates a feature detection service, capable of monitoring the sensor data for significant events (e.g. irregular ECG behaviour). If atypical features are detected, GRID notification services are used to warn the clinician monitoring the patient.

\subsection{Integration}

All sensors are auto-detected. When a sensor is pluggedin, the system discovers the sensor, cache sensor output until a network connection becomes available, and subsequently make the sensor output available on the grid. In the case of simple sensors such as ECG or $\mathrm{SpO} 2$, the data can just be viewed on the grid. In the case of more complicated data (such as location data provided by a GPS sensor), a GRID client may have to be developed for that sensor. In the case of the GPS data, the client may have to perform a map lookup to identify which speed the patient is walking at, and which gradient the hill has.

\section{Conclusion}

We have presented a modular diagnostic monitor, built around a wearable which is connected to the GRID via a wireless network. Modularity allows both researchers and practitioners to utilise various sensor modules, reconfigure the unit in terms of its wireless telemetry capabilities, select the appropriate level of data preprocessing (before archiving data) and choose the appropriate level and nature of feedback to the subject. Once on the GRID, the data is stored in a database, and can be used for a live display, viewing historical data or data mining. We have demonstrated our system, and are at present looking into trialling the system.

\section{Acknowledgements}

We are grateful to $3 \mathrm{M}$, who provided us with the electrodes for the ECG sensor.

\section{References}

[1] C. Barratt, B. Hayes-Gill, J. Crowe, J. Skoyles, and J. Campbell. Telemetric measurement of surface skin temperature during cardiopulmonary bypass. In Proc. of European Medical \& Biological Engineering Conference, pages 554-555,
Vienna, Austria, November 1999. Med.Biol.Eng.Comp., 37, Supplement 2, Part 1.

[2] C. Barratt, B. Hayes-Gill, H. Vyas, and J. Crowe. Selection of pulse oximetry equipment for ambulatory monitoring. Journal Medical Eng \& Tech, 21(1):17-24, 2001.

[3] S. Benford, N. Crout, J. Crowe, S. Egglestone, M. Foster, C. Greenhalgh, A. Hampshire, B. Hayes-Gill, J. Humble, A. Irune, J. Laybourn-Parry, B. Palethorpe, T. Reid, and M. Sumner. e-Science from the antarctic to the GRID. Escience all hands meeting.

[4] J. Crowe, A. Harrison, and B. Hayes-Gill. The feasibility of long-term fatal heart rate monitoring in the home environment using maternal abdominal electrodes. Physiol. Meas, 16:195-202, 1995.

[5] J. Crowe, B. Hayes-Gill, B. Francon, L. Hardebecke, D. Rogers, Y. Thong, P. Dimmock, K. Wyatt, and P. O'Brien. Customisation of a personal digital assistant for logging premenstrual syndrome symptoms. British Journal of Healthcare Computing \& Information Management, 17(4):33-35, 2000.

[6] J. Echevarria, M. Woolfson, J. Crowe, and B. Hayes-Gill. Interpretation of heart rate variability data via detrended fluctuation analysis and alpha beta filter. Chaos, 13:467-475, 2003.

[7] GLOBUS. The globus toolkit. http://www.globus .org/toolkit/, April 2003.

[8] J. Hall and J. Crowe. Ambulatory electrocardiogram compression using wavelet packets to approximate the Karhunen-Loeve transform. Applied Signal Processing, 3:25-36, 1996.

[9] A. Harrison, B. Hayes-Gill, J. Crowe, and S. Chang. The application of an Actel field programmable gate array in the design of an ecg rr interval recorder. Journal Medical Eng. and Tech, 19(6):198-204, 1995.

[10] OpenGIS Consortium Inc. Sensor modelling language (SensorML). http://vast.uah.edu/SensorML/OGC -02-026_SensorML_0 . 07r4 .pdf, December 2002.

[11] J. Pieri, J. Crowe, B. Hayes-Gill, C. Spencer, K. Bhogal, and D. James. Compact long-term recorder of the transabdominal foetal and maternal electrocardiogram. Med.Biol.Eng. \& Comp., 39(1):118-125, 2001.

[12] C. Randell and H. L. Muller. The well mannered wearable computer. Personal and Ubiquitous Computing, 6(1):31-36, February 2002.

[13] J. Taylor, B. Hayes-Gill, J. Crowe, and C. Paull. Towards multi-patient leadless and wireless cardiotocography via RF telemetry. Med.Eng. \& Physics, 20:764-772, 1998.

[14] The Open Grid Services Architecture Working Group (OGSA WG). Open GRID services architecture: A roadmap. http : / /www.ggf .org/ogsa-wg/ogsa_roadmap. 0 . 4 .pdf, December 2002.

[15] Environmental monitoring using the GRID. http : / / www . gridoutreach.org.uk/docs/pilots/equator .htm, 2002.

[16] GRID based medical devices for everyday health. http://www.gridoutreach.org.uk/docs/pilo ts/meddev. htm, 2002. 